\title{
Mobile Small-cell Deployment Strategy for Hot Spot in Existing Heterogeneous Networks
}

\author{
Nan E, Xiaoli Chu and Jie Zhang \\ Department of Electronic and Electrical Engineering, University of Sheffield \\ Sheffield, S1 3JD, United Kingdom \\ nan.e@sheffield.ac.uk, x.chu@sheffield.ac.uk, jie.zhang@sheffield.ac.uk
}

\begin{abstract}
As the mobile data demand keeps growing, an existing heterogeneous network (HetNet) composed of macrocells and small cells may still face the problem of not being able to provide sufficient capacity for unexpected but reoccurring hot spots. In this paper, we propose a mobile small-cell deployment strategy that avoids replanning the overall network while fulfilling the hot spot demand by optimizing the deployment of additional mobile small cells on top of the existing HetNet. We formulate the problem as a joint optimization over the number and locations of mobile small cells and the user associations of all cells in order to maximize the minimum user throughput. In order to solve it, we first propose a Fixed Number Deployment Algorithm (FNDA) to solve the problem with a fixed number of new small cells. Afterwards, we extend FNDA into a Deployment Over Existing Network Algorithm (DOENA) to solve the joint optimization problem. The simulation results show that DOENA offers a higher minimum user throughput while requiring less mobile small cells to be deployed than the deployment optimization based on maximizing sum user throughput.
\end{abstract}

Index Terms-HetNet, small cell deployment, optimization

\section{INTRODUCTION}

$\mathbf{H}$ ETEROGENEOUS networks (HetNets) as an important concept in LTE-A is widely used by operators and network designers nowadays. It is anticipated that HetNets would mitigate the conflict between the rapid growth of data demand and limited radio resources by increasing the area spectral efficiency through densely deploying low-power small cells such as femtocells [1]. For example, a HetNet can be constructed by overlaying low-power small base stations (BSs) on top of the existing macrocell network to increase the network capacity [2].

A HetNet is usually planned based on the expected user distribution and mobile traffic pattern obtained from longterm observations and big data collections. It is important to achieve both good service quality and low cost in HetNet planning and deployment. In [3], the deployment of small cells is optimized to obtain the best tradeoff between user Quality of Service (QoS) and operators' costs. The dynamic small cell deployment strategy in [4] can be used to find out when and where small cells need to be deployed.

Once a HetNet has been deployed, its radio resources need to be reused among neighboring cells and the network capacity is limited by inter-cell interference. In [5], interference management is achieved by controlling the number of resource blocks (RBs) that can be used by small cells. In [6], the resource allocation and user association strategies

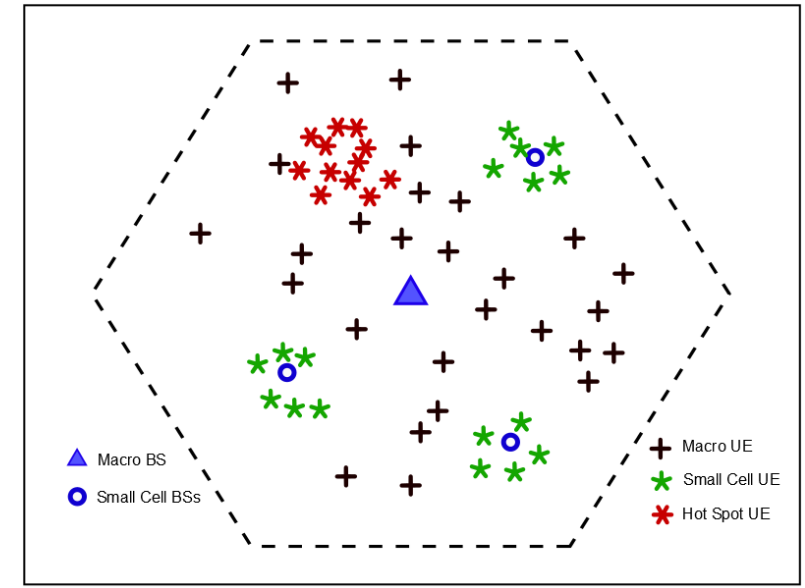

Fig. 1: An instance of the problem scenario.

are investigated for the orthogonal deployment, co-channel deployment and partially shared deployment of small cells.

However, in an existing HetNet, persistent clusters of user equipments (UEs), a.k.a., hot spots (HSs), which were not been expected in the original network planning may occur, causing extra traffic demand. When the mobile traffic demand goes beyond the network capacity, additional small cells may need to be deployed on top of the existing HetNet. In this case, the strategies in [3] [4] [7] and the structured deployment strategy in [8] would require the redesign of the overall HetNet to achieve the optimized deployment. In [9], the optimized deployment locations of new small cells are selected from a set of candidate locations, which need to be obtained before the optimization process, making it less effective for unexpected but reoccurring HSs. Moreover, without constraint of minimum UE throughput, maximizing the sum throughput or average UE throughput [10] might still leave some UEs with dissatisfied QoS.

In this paper, we propose a strategy to optimize the number and locations of additional mobile small cells on top of an existing HetNet to fulfil the excess traffic demand of recurring HSs that were not expected in the original HetNet planning. The mobile small cells can be mounted on vehicles so that they can be deployed by the operator in response to the HSs in a timely manner. It is assumed that the number and locations of the HS UEs are statistically known by the operator. Since the time scales of deploying mobile small cells and user association are much longer than that of resource allocation, 
we assume round-robin and even resource allocation to UEs in each cell for simplicity. We propose to maximize the minimum UE throughput through a joint optimization of the number and locations of additional mobile small cells and the user associations of all cells. The simplified optimization problem is still NP-hard. Hence, we first propose the Fixed Number Deployment Algorithm (FNDA) to optimize the locations of mobile small cells and user associations for a given number of mobile small cells. Afterwards, we extend FNDA into the Deployment over Existing Network Algorithm (DOENA) to jointly optimize the number and locations of mobile small cells deployment together with the user associations of all cells. Performance of the proposed DOENA is evaluated in terms of minimum user throughput and number of mobile small cells required through simulations. The relationship between the number of required mobile small cells and the number of HS UEs is discussed. The comparison between deployments from maximizing minimum UE throughput and maximizing sum UE throughput [10] is also included in the simulation.

The rest of the paper is organized as follows. In Section II, the system model is presented. The formulation of the optimization problem and its transformations are provided in Section III. The FNDA and DOENA are provided in Section IV and simulation results and performance evaluation are presented in Section V. Conclusions are drawn in Section VI.

\section{SySTEM MODEL}

We consider the downlink (DL) of a two-tier HetNet consisting of one central macrocell and $N_{F}$ small cells, all sharing the same spectrum. With one HS randomly distributed in the macrocell coverage area, all non-HS UEs are uniformly distributed within the coverage area of their serving cell, and the HS UEs are uniformly distributed in the HS area. The coverage area of each macrocell or each small cell is assumed to be a disk area centred at the macro or small BS with a certain radius. The HS area is also assumed to be circular for simplicity. Denote the coverage area of the macrocell as $\mathcal{H}$. The total number of existing BSs is $N_{e B S}=1+N_{F}$. Denote the number of mobile small cells to be deployed as $N_{n B S}$, and the total number of all BS would be $N_{B S}=N_{e B S}+N_{n B S}$. Each cell has access to the total of $N_{R B}$ resource blocks (RBs). Let $N_{n h s}$ denote the number of non-HS UEs and $N_{h s}$ denote the number of not expected but reoccurring HS UEs. The total number of UEs is given by $N_{U}=N_{n h s}+N_{h s}$. The set of all UEs is denoted as $\mathcal{N}_{U}=\left\{1,2, \ldots, N_{U}\right\}$, the set of BSs is denoted as $\mathcal{N}_{B S}=\left\{1,2, \ldots, N_{B S}\right\}$ and the set of RBs is denoted as $\mathcal{N}_{R B}=\left\{1,2, \ldots, N_{R B}\right\}$.

The throughput of the $i$ th UE is given by:

$$
\gamma_{i}=\sum_{k=1}^{N_{R B}} \sum_{j=1}^{N_{B S}} B \cdot \log _{2}\left(1+\frac{P_{j}^{k} \cdot g_{i, j}^{k} \cdot a_{i, j}^{k}}{I_{i, j}^{k}+N_{0}}\right),
$$

where $a_{i, j}^{k}=1$ if the $i$ th UE is served by the $j$ th BS in the $k$ th RB, $a_{i, j}^{k}=0$ otherwise; $B$ is the bandwidth of a RB, $P_{j}^{k}$ is the DL transmit power of the $j$ th $\mathrm{BS}$ in the $k$ th $\mathrm{RB}, g_{i, j}^{k}$ is the channel power gain of the link between the $i$ th UE and the $j$ th $\mathrm{BS}$ in the $k$ th $\mathrm{RB}$ and can be expressed as:

$$
g_{i, j}^{k}=g_{f, i j}^{k} \cdot g_{p l, i j}
$$

where $g_{f, i j}^{k}$ is the exponentially distributed fading gain with unit mean, and $g_{p l, i j}$ is the pathloss gain given by [11]:

$$
g_{p l, i j}=-15.3-\alpha \cdot 10 \log _{10}\left(\sqrt{\left(x_{i}-x_{j}\right)^{2}+\left(y_{i}-y_{j}\right)^{2}}\right) \mathrm{dB}
$$

where $\left(x_{i}, y_{i}\right)$ are the location coordinates of the $i$ th UE and $\left(x_{j}, y_{j}\right)$ are the coordinates of the $j$ th $\mathrm{BS} ; \alpha$ is the path loss distance exponent; $N_{0}$ is the additive white Gaussian noise (AWGN) power; and $I_{i, j}^{k}$ is the interference power received by $\mathrm{UE} i$ in the $k$ th RB from BSs other than BS $j$ i.e.,

$$
I_{i, j}^{k}=\sum_{\substack{i^{\prime}=1,1, j^{\prime}=1, i^{\prime} \neq i \\ j^{\prime} \neq j}}^{N_{U}} P_{j^{\prime}}^{k} \cdot g_{i, j^{\prime}}^{k} \cdot a_{i^{\prime}, j^{\prime}}^{k}
$$

\section{Mobile Small Cell Deployment Optimization}

\section{A. Joint Optimization}

The joint optimization is defined as maximizing the minimum UE throughput among all UEs over the number and locations of mobile small cells, user associations of all cells, and resource allocation in each cell. That is,

$$
\begin{aligned}
& \underset{\mathbf{A},(\mathbf{x}, \mathbf{y})}{\arg \max } \min _{i}\left\{\gamma_{i}\right\}, i \in \mathcal{N}_{U} . \\
& \text { s.t. } \gamma_{i}>\gamma_{t h}, \forall i \in \mathcal{N}_{U} . \\
& \qquad(\mathbf{x}, \mathbf{y}) \in|\mathcal{H}| . \\
& \sum_{j=1}^{N_{B S}} \min \left(\sum_{k=1}^{N_{R B}} a_{i j}^{k}, 1\right)=1, \forall i \in \mathcal{N}_{U} . \\
& \sum_{k=1}^{N_{R B}} a_{i j}^{k} \leq N_{R B}, \forall i \in \mathcal{N}_{U}, j \in \mathcal{N}_{B S} . \\
& \sum_{i=1}^{N_{U}} a_{i j}^{k} \leq 1, \forall j \in \mathcal{N}_{B S}, k \in \mathcal{N}_{R B} . \\
& a_{i, j}^{k} \in\{0,1\}, \forall i \in \mathcal{N}_{U}, \forall j \in \mathcal{N}_{B S}, k \in \mathcal{N}_{R B} .
\end{aligned}
$$

where $\mathbf{A}$ is the $N_{U} \times N_{B S} \times N_{R B}$ matrix that contains all $a_{i, j}^{k}$ as elements, $(\mathbf{x}, \mathbf{y})$ are the coordinate vectors that contain locations of all mobile small cells, $\mathbf{x}$ and $\mathbf{y}$ are each of size $N_{n B S} \times 1, \gamma_{i}$ is the throughput of the $i$ th UE as given in (1), without loss of generality, we denote the macro BS as the first BS (i.e., $j=1$ ), (6) guarantees that each UE throughput is beyond the threshold $\gamma_{t h}$, (7) limits the deployment area for small cells with respect to the macro BS, $|\mathcal{H}|$ is the feasible deployment area for mobile small cells as presented in [12] with the exclusion of coverage overlap between any two small cells. (8) guarantees that each UE is served by one BS, (9) limits the number of RBs each UE can be allocated, (10) ensures that each RB can be allocated to at most one UE in each cell, and (11) is the binary indicator constraint. 


\section{B. Decomposed Optimization}

In (5), $\mathbf{A}$ is a UE-to-BS and UE-to-RB joint association matrix. The joint optimization over the number and locations of mobile small cells, user association to all cells, and resource allocations per cell has a very high complexity. Therefore, we decompose the joint association matrix into two matrixes: 1) user association matrix, and 2) resource allocation matrix. Accordingly, the joint association indicator $a_{i, j}^{k}$ is given by:

$$
a_{i, j}^{k}=b_{i, j} \cdot c_{i, k}
$$

where $b_{i, j}=1$ if UE $i$ is served by BS $j$, otherwise $b_{i, j}=0$; $c_{i, k}=1$ if UE $i$ is allocated with RB $k$, otherwise $c_{i, k}=0$.

The throughput of the $i$ th UE can be rewritten as:

$$
\gamma_{i}=\sum_{k=1}^{N_{R B}} \sum_{j=1}^{N_{B S}} B \cdot \log _{2}\left(1+\frac{P_{j}^{k} \cdot g_{i, j}^{k} \cdot b_{i, j} \cdot c_{i, k}}{I_{i, j}^{k}+N_{0}}\right)
$$

The interference power received by the $i$ th UE that is served by $\mathrm{BS} j$ in the $k$ th $\mathrm{RB}$ can be rewritten as:

$$
I_{i, j}^{k}=\sum_{\substack{i^{\prime}=1, i^{\prime} \neq i}}^{N_{U}} \sum_{\substack{j^{\prime}=1, j^{\prime} \neq j}}^{N_{B S}} P_{j^{\prime}}^{k} \cdot g_{i, j^{\prime}}^{k} \cdot b_{i^{\prime}, j^{\prime}} \cdot c_{i^{\prime}, k}
$$

The decomposed optimization problem is defined as:

$$
\begin{gathered}
\underset{\mathbf{B}, \mathbf{C},(\mathbf{x}, \mathbf{y})}{\arg \max } \min _{i}\left\{\gamma_{i}\right\}, i \in \mathcal{N}_{U} . \\
\text { s.t. (6), (7), } \sum_{j=1}^{N_{B S}} b_{i, j}=1, \forall i \in \mathcal{N}_{U} . \\
\sum_{k=1}^{N_{R B}} c_{i, k} \leq N_{R B}, \forall i \in \mathcal{N}_{U} . \\
\sum_{i=1}^{N_{U}} c_{i, k} \cdot b_{i, j} \leq 1, \forall j \in \mathcal{N}_{B S}, k \in \mathcal{N}_{R B} . \\
b_{i, j}, c_{i, k} \in\{0,1\}, \forall i \in \mathcal{N}_{U}, \forall j \in \mathcal{N}_{B S}, k \in \mathcal{N}_{R B} .
\end{gathered}
$$

where $\mathbf{B}$ is the $N_{U} \times N_{B S}$ UE-to-BS association matrix that contains all $b_{i, j}$ as elements, $\mathbf{C}$ is the $N_{U} \times N_{R B}$ UE-to$\mathrm{RB}$ association matrix that contains all $c_{i, k}$ as elements, (16) guarantees that each UE is served by one BS, (17) guarantees that the number of RBs allocated to each UE is not beyond the total available RBs, (18) guarantees that each RB is allocated to at most one UE in each cell, and (19) is the binary indicators constraint.

\section{Simplified Optimization for Mobile Small-cell Deployment}

The deployment of additional mobile small cells is for meeting the excessive data demand in the period of HS occurrence, during which the HS UEs' locations are relatively stable. In contrary to resource allocation, the deployment optimization of mobile small cells and user associations do not need to be updated frequently in this situation. Hence, we propose to optimize the number and locations of mobile small cells by solving for the matrix $\mathbf{B}$ under the following assumptions.
Assumption 1: We assume that the RBs are allocated in each cell following the round-robin algorithm with full bandwidth allocation [13]. That is, all the $N_{R B}$ RBs are allocated to UEs in each cell following the round robin algorithm at all times, and there will be inter-cell interference in each RB. This can be considered as the worst-case interference scenario.

The number of RBs allocated by the $j$ th BS to the $i$ th UE can be calculated as:

$$
N_{i, j}^{R B}=b_{i, j} \sum_{k=1}^{N_{R B}} c_{i, k}, \forall i \in \mathcal{N}_{U}, j \in \mathcal{N}_{B S}
$$

Without loss of generality, we assume that the total number of RBs are evenly distributed among the UEs in each cell, and relax the constraint that the number of RBs per UE has to be an integer. Accordingly, the number of RBs per UE in cell $j$ can be written as:

$$
N_{j}^{R B}=\frac{N_{R B}}{\sum_{i=1}^{N_{U}} b_{i, j}}, \forall j \in \mathcal{N}_{B S}
$$

Assumption 2: Given the relatively long time scales of small cell deployment and user association, we assume that the effect of fast fading has been averaged out.

Since we do not consider power control in the DL, a BS uses the same transmit power in all RBs and $P_{j}^{k}$ is expressed as:

$$
P_{j}^{k}=P_{j}
$$

where $P_{j}$ is the DL transmit power per RB of the $j$ th $\mathrm{BS}$. If the DL transmit power per RB of a macro BS and a small BS is given by $P_{M}$ and $P_{F}$, respectively, then $P_{1}=P_{M}$, and $P_{j}=P_{F}$ for $j=2,3, \ldots, N_{B S}$.

In this case, we can ignore the superscript $k$ hereafter and the UE throughput $\gamma_{i}$ can be rewritten as:

$$
\gamma_{i}=\sum_{j=1}^{N_{B S}} N_{j}^{R B} \cdot B \cdot \log _{2}\left(1+\frac{P_{j} \cdot g_{p l, i j} \cdot b_{i, j}}{I_{i, j}+N_{0}}\right)
$$

Accordingly, the interference power received by UE $i$ in an $\mathrm{RB}$ when it is served by $\mathrm{BS} j$ is given by

$$
I_{i, j}=\sum_{j^{\prime}=1, j^{\prime} \neq j}^{N_{B S}} P_{j^{\prime}} \cdot g_{p l, i j^{\prime}}
$$

Thus, the mobile small-cell deployment optimization problem can be simplified as:

$$
\underset{\mathbf{B},(\mathbf{x}, \mathbf{y})}{\arg \max } \min _{i}\left\{\gamma_{i}\right\}, i \in \mathcal{N}_{U} .
$$

$$
\begin{gathered}
\text { s.t. (6), (7), } \sum_{j=1}^{N_{B S}} b_{i, j}=1, \forall i \in \mathcal{N}_{U} . \\
N_{R B} \geq N_{j}^{R B}>0, \forall j \in \mathcal{N}_{B S} \\
b_{i, j} \in\{0,1\}, \forall i \in \mathcal{N}_{U}, \forall j \in \mathcal{N}_{B S} .
\end{gathered}
$$

where (27) guarantees that the number of RBs each UE can be allocated is not beyond the total number of available RBs. 


\section{Solving the Optimization Problem}

In (25), the size of vectors $\mathbf{x}$ and $\mathbf{y}$, i.e., the number $N_{n B S}$ of mobile small cells to be deployed, is also an unknown variable to be determined. Note that the joint optimization of the number and locations of mobile small cells together with the user association in (25) is a mixed integer programming problem, which is NP-hard. The difficulty of finding the global optimal solution is high due to the computational complexity. In this section, we first propose a simple algorithm to solve the optimization problem (25) for a given feasible number of mobile small cells, then we extend it to an algorithm to solve the joint optimization problem in (25).

\section{A. Fixed Number Mobile Small-cell Deployment Algorithm}

Given a feasible value of the number of mobile small cells to be deployed $N_{n B S}$, the objectives of (25) are reduced to finding the deployment locations of mobile small cells and user associations of all cells. In order to solve this simplified problem, we propose a Fixed Number Deployment Algorithm (FNDA) based on the branch and bound (B\&B) method, where the binary constraint of user association indicators is relaxed to $0 \leq b_{i, j} \leq 1[14]$.

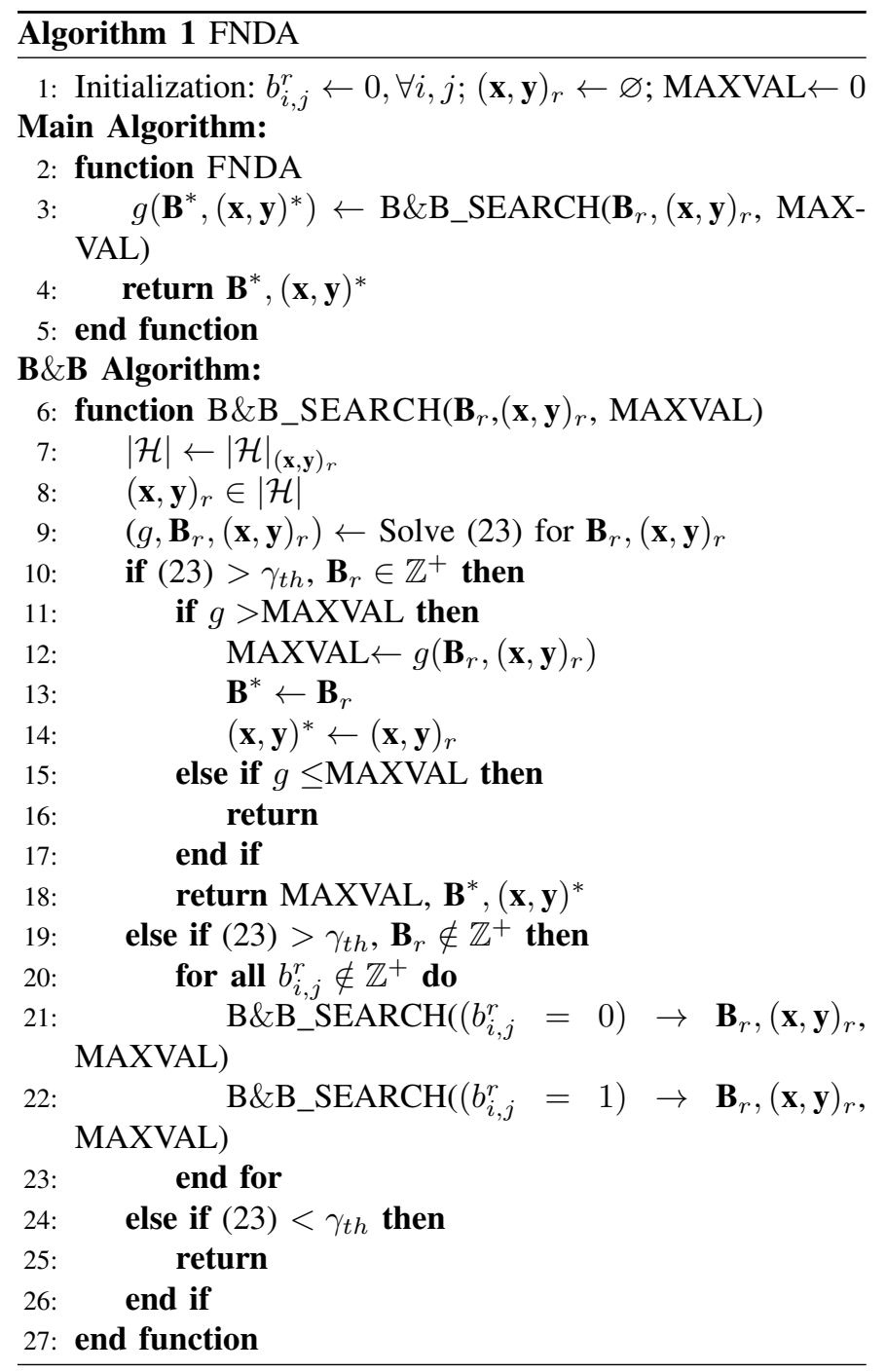

Denote $g$ as the minimum UE throughput calculated by (23), $\mathbf{B}_{r}$ as the user association matrix with all elements relaxed to $0 \leq b_{i, j}^{r} \leq 1$, and $(\mathbf{x}, \mathbf{y})_{r}$ as the location vectors of mobile small cells each with known size of $N_{n B S}$. The FNDA is presented in Algorithm 1.

The optimal solutions of mobile small cells' deployment locations and user associations will be returned in $\mathbf{B}^{*}$ and $(\mathbf{x}, \mathbf{y})^{*}$, respectively.

\section{B. Mobile Small-cell Deployment over Existing Network Al- gorithm}

For a given operator, there would usually be a maximum number of mobile small cells $N_{n B S}^{\max }$ that can be deployed due to cost and infrastructure considerations. We define the set $\mathbf{S}=$ $\left\{s_{j}\right\}$ containing all existing BSs and the maximum number of mobile small cells. The size of $\mathbf{S}$ is $N_{B S}^{\max }=N_{e B S}+N_{n B S}^{\max }$. If BS $j$ has been deployed or is to be deployed, then $s_{j}=1$; otherwise, $s_{j}=0$. Obviously, $\forall j \in\left\{1,2, \ldots, N_{e B S}\right\}, s_{j}=1$. The set of maximum number of mobile small cells is denote as $\mathcal{N}_{n B S}^{\max }=\left\{N_{e B S}, \ldots, N_{e B S}+N_{n B S}^{\max }\right\}$.

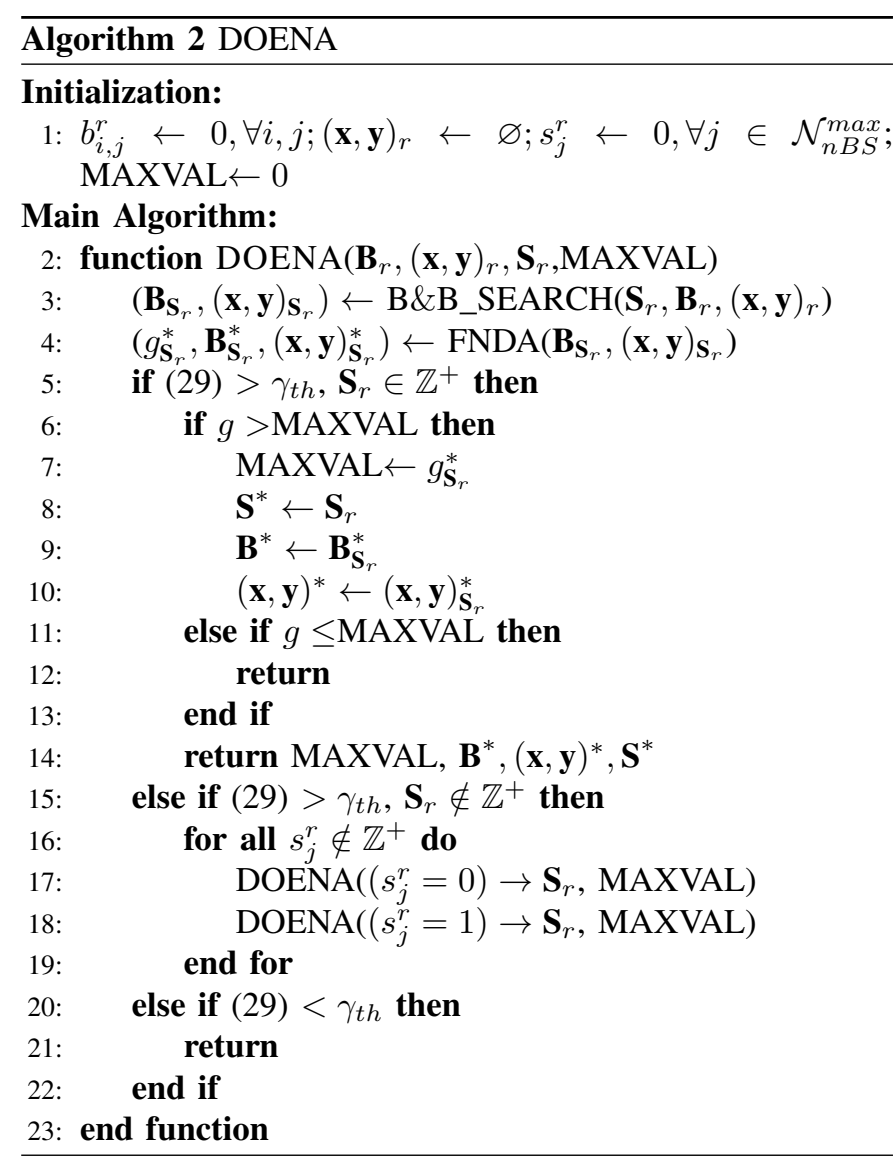

Accordingly, (23) can be rewritten as:

$$
\gamma_{i}=\sum_{j=1}^{N_{B S}^{\max }} s_{j} \cdot N_{j}^{R B} \cdot B \log _{2}\left(1+\frac{P_{j} \cdot g_{p l, i j} \cdot b_{i, j}}{I_{i, j}+N_{0}}\right)
$$

In order to solve the joint optimization in (25), we relax the binary constraints on both $b_{i, j}$ and $s_{j}$ to $0 \leq b_{i, j} \leq 1$ and 


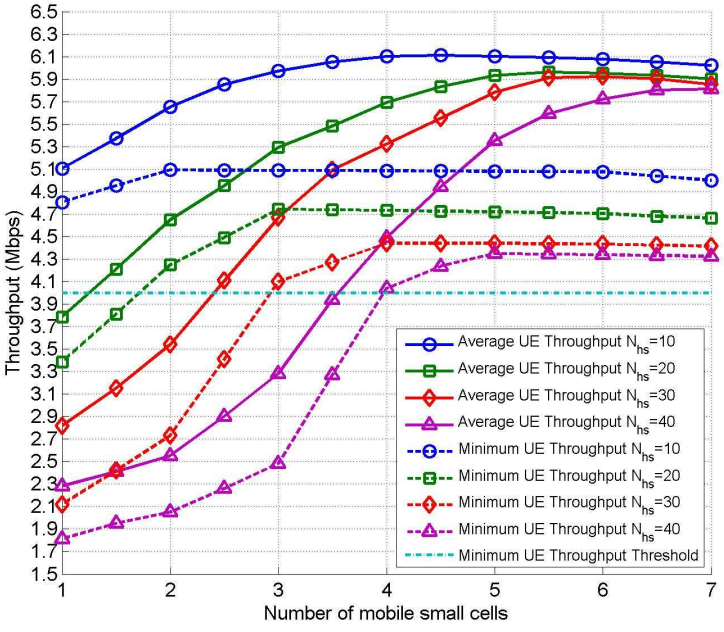

Fig. 2: The average UE throughput and minimum UE throughput versus the number of mobile small cells by FNDA.

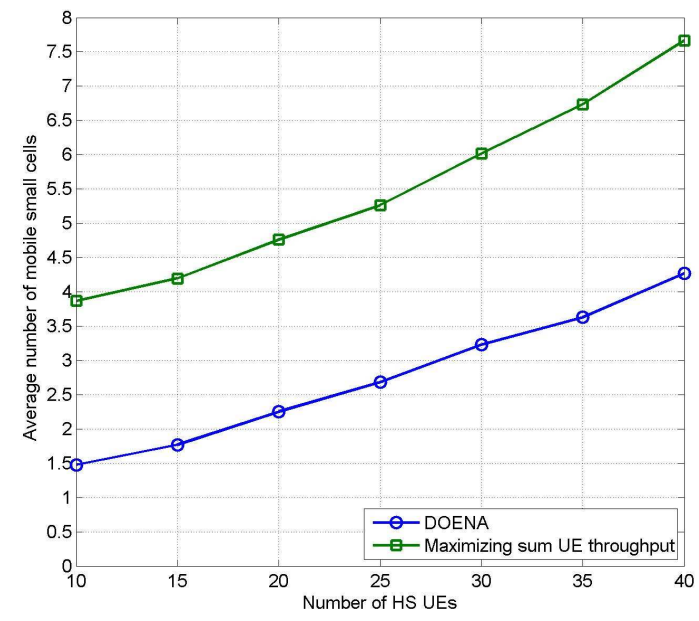

Fig. 3: The average number of mobile small cells required by DOENA and by the deployment optimization based on maximizing sum UE throughput versus the number of HS UEs.

$0 \leq s_{j} \leq 1$, and put the relaxed indicators in matrixes $\mathbf{B}_{r}$ and $\mathbf{S}_{r}$, respectively. The DOENA is presented in Algorithm 2.

Based on the returned value $\mathbf{S}^{*}$, the optimal number of mobile small cells can be calculated by:

$$
N_{n B S}=\sum_{j=N_{e B S}+1}^{N_{B S}^{\max }} s_{j}^{*}
$$

The mobile small cells' deployment locations and user associations will be returned in $(\mathbf{x}, \mathbf{y})^{*}$ and $\mathbf{B}^{*}$, respectively.

\section{Simulation Results}

In this section, we present simulation results to evaluate the performance of the proposed deployment strategy of mobile small cells. In each run of the simulation, the $N_{F}$ existing small cells are independently and uniformly distributed in the coverage area of the macrocell, the location of the HS

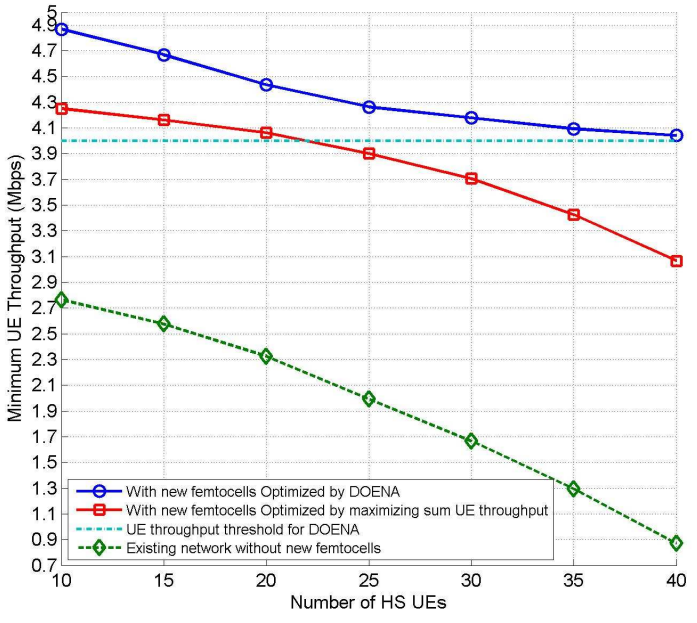

Fig. 4: The minimum UE throughput of DOENA and maximizing sum UE throughput versus the number of HS UEs.

is randomly generated within the macrocell coverage area following a uniform distribution as well, and the locations of all UEs are randomly generated following the system model in Section II. The system parameters used in our simulation are given in Table I.

The minimum UE throughput and average throughput achieved by the proposed FNDA versus the number of mobile small cells are shown in Fig. 2. We can see that both average UE throughput and minimum UE throughput increase with the increasing number of mobile small cells, and can exceed the UE throughput threshold when the number of mobile small cells is sufficiently large for a given number of HS UEs. However, as the number of mobile small cells goes beyond a certain value, the increase of UE throughput slows down, and the minimum UE throughput even starts to decrease. This is because, the inter-cell interference becomes dominant and diminishes the capacity gain offered by dense deployment of small cells.

Fig. 3 shows the average number of mobile small cells required by the proposed DOENA and by the small cel1 deployment optimization based on maximizing sum UE throughput [10] versus the number of HS UEs, under the same minimum UE throughput constraint. Since the scheme based

TABLE I: System Setting

\begin{tabular}{|c|c|}
\hline Parameter & Value \\
\hline$P_{M}$ & $46 \mathrm{dBm}$ \\
$P_{F}$ & $23 \mathrm{dBm}$ \\
$N_{n h s}$ & 50 \\
$N_{h s}$ & {$[10,40]$} \\
$N_{e B S}$ & 4 \\
$N_{F}$ & 3 \\
$N_{n B S}^{m a x}$ & 10 \\
$N_{R B}$ & 50 \\
$\gamma_{t h}$ & $4 \mathrm{Mbps}$ \\
Macrocell Radius & $300 \mathrm{~m}$ \\
Small cell Radius & $20 \mathrm{~m}$ \\
HS Radius & $60 \mathrm{~m}$ \\
$N_{0}$ & $-174 \mathrm{~dB} / \mathrm{Hz}$ \\
$\alpha$ & 4 \\
\hline
\end{tabular}




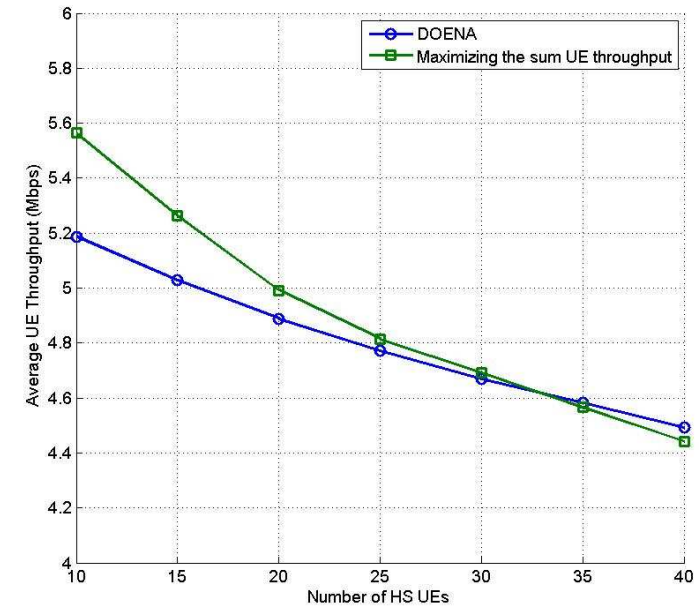

Fig. 5: The average UE throughput of DOENA and maximizing sum UE throughput versus the number of HS UEs.

on maximizing sum throughput in [10] optimizes the number and locations of all small cells in an iterative manner for given user association, for a fair comparison in the simulation we optimize its user association using the same method as in DOENA and initialize the iteration with the randomly generated locations of existing small cells. We can see that the optimal number of mobile small cells for maximizing sum UE throughput is larger than that from DOENA for all considered numbers of HS UEs. Deploying less mobile small cells while fulfilling the extra mobile traffic demand of HS UEs will reduce the capital and operational costs of the operator.

Fig. 4 plots the minimum UE throughput versus the number of HS UEs before and after the deployment of mobile small cells for DOENA and for maximizing sum UE throughput. It shows that as the number of HS UEs increases, if without mobile small cells, the minimum UE throughput falls below the UE throughput threshold and decreases significantly, indicating that the existing HetNet can no longer fulfil the total traffic demand. The minimum UE throughput of the DOENA is higher than that of the deployment optimized by maximizing the sum UE throughput, and it can be kept above the threshold even for a large number of HS UEs. However, with the number of HS UEs increasing, the minimum UE throughput of deployment optimized by maximizing the sum UE throughput falls below the threshold, which indicates that the QoS of some UEs cannot be satisfied.

Fig. 5 plots the average UE throughput achieved by DOENA and by small cell deployment optimization based on maximizing sum UE throughput versus the number of HS UEs. We can see that the deployment optimized by maximizing sum UE throughput offers a higher average UE throughput than DOENA when the number of HS UEs is low, but its average UE throughput decreases faster with the number of HS UEs than DOENA. As a result, DOENA outperforms the deployment optimization based on maximizing sum UE throughput in terms of average UE throughput (and sum UE throughput) when the number of HS UEs is large.

\section{CONCLUSION}

In this paper, we have proposed a deployment strategy of additional mobile small cells on top of an existing HetNet in order to fulfil the extra traffic demand of recurring HSs that have not been considered in the original network planning. The optimization problem is first formulated as maximizing the minimum UE throughput jointly over the number and locations of mobile small cells, user associations of all cells, and resource allocation in each cell. We then simplified the problem into a joint optimization of mobile small cell deployment and user association. By solving the simplified optimization problem, we first proposed the FNDA to optimize the deployment locations of mobile small cells and the user association for a given feasible number of mobile small cells. Based on FDNA, we then proposed the DOENA algorithm to jointly optimize the number and locations of mobile small cells together with user association. The simulation results have shown that compared with the deployment optimization based on maximizing sum UE throughput, the proposed DOENA offers higher minimum UE throughput while requiring less mobile small cells to be deployed, and higher average UE throughput when the number of HS UEs is large.

\section{REFERENCE}

[1] 3GPP TR 36.912 V2.0.0, "3GPP; technical specification group radio access network; feasibility study for further advancements for eutra (release 9)," August 2009.

[2] A. Khandekar, N. Bhushan, J. Tingfang, and V. Vanghi, "LTE-advanced: Heterogeneous networks," in Wireless Conference, 2010, pp. 978-982.

[3] H. Y. Hsieh, S. E. Wei, and C. P. Chien, "Optimizing small cell deployment in arbitrary wireless networks with minimum service rate constraints," IEEE Transactions on Mobile Computing,, vol. 13, no. 8, pp. 1801-1815, Aug 2014.

[4] M. Qutqut, H. Abou-zeid, H. Hassanein, A. Rashwan, and F. AlTurjman, "Dynamic small cell placement strategies for LTE heterogeneous networks," in IEEE Symposium on Computers and Communication, June 2014, pp. 1-6.

[5] D. K. Shin, W. Choi, and T. Yu, "Statistically controlled opportunistic resource block sharing for femto cell networks," Journal of Communications and Networks, vol. 15, no. 5, pp. 469-475, Oct 2013.

[6] D. Fooladivanda and C. Rosenberg, "Joint resource allocation and user association for heterogeneous wireless cellular networks," Trans. Wireless Comm., vol. 12, no. 1, pp. 248-257, Jan 2013.

[7] W. Zhao, S. Wang, C. Wang, and X. Wu, "Cell planning for heterogeneous networks: An approximation algorithm," in INFOCOM 2014, April 2014.

[8] R. Razavi and H. Claussen, "Urban small cell deployments: Impact on the network energy consumption," in Wireless Communications and Networking Conference Workshops, April 2012, pp. 47-52.

[9] I. Siomina and D. Yuan, "Optimization approaches for planning small cell locations in load-coupled heterogeneous lte networks," in IEEE PIMRC, Sep 2013, pp. 2904-2908.

[10] Y. Park, J. Heo, H. Kim, H. Wang, S. Choi, T. Yu, and D. Hong, "Effective small cell deployment with interference and traffic consideration," in IEEE VTC Fall, Sept 2014, pp. 1-5.

[11] 3GPP TR 36.921 v11.0.0, "E-UTRA FDD Home eNode B Radio Frequency requirements analysis (Release 11)," October 2012.

[12] J. Wu, X. Chu, D. Lopez-Perez, and H. Wang, "Femtocell exclusion regions in hierarchical 3-sector macrocells for co-channel deployments," in IEEE International Conference on Communications in China, Aug 2012, pp. 541-545.

[13] 3GPP TR 36.814 V9.0.0, "Evolved Universal Terrestrial Radio Access (E-UTRA); Further advancements for E-UTRA physical layer aspects (Release 9)," March 2010.

[14] J. M. Ortega and W. C. Rheinboldt, Iterative solution of nonlinear equations in several variables. Siam, 1970, vol. 30. 